\title{
An Ontology-based Framework and its Application to Effective Collaboration
}

\author{
Seiji Isotani and Riichiro Mizoguchi \\ The Institute of Scientific and Industrial Research, Osaka University \\ 8-1 Mihogaoka, Ibaraki, Osaka, Japan \\ isotani@acm.org,miz@ei.sanken.osaka-u.ac.jp
}

\begin{abstract}
In the past few years Artificial Intelligence has been gradually introduced to enhance Education through technologies. However, usual approaches provide systems with a kind of expertise using a set of heuristics and domain theories built in the procedures. As a result they cannot justify their recommendations systematically and scientifically. To overcome such problems it is necessary to establish a common understanding of what a learning theory is and how to represent it adequately. In this work we present part of our ontological framework that allows for the partial representation of learning theories considering explicitness, formalism, concepts and vocabulary. Then, we propose sophisticated techniques to reasoning on these theories considering their semantics showing the use of this framework to build a system called CHOCOLATO to facilitate the effective design and analysis of collaborative learning activities.
\end{abstract}

Keywords: Ontological engineering, CSCL, theory-aware system

\section{Introduction}

Two of the most important research subjects during the development of intelligent educational systems (IES) are the modeling of knowledge and the extraction of knowledge flows from theory to practice [4; $8 ; 15]$. It bridges the gap between theoretical understanding about learning and the practical foundations to support the learning process through the use of technology. To facilitate the representation and extraction of knowledge in educational systems, Artificial Intelligence techniques have been gradually and successfully introduced in the field. However, major challenges still remain. Among these, we are interested in how to represent semantically the knowledge of intelligent educational systems (IES) and then how to use this knowledge efficiently, especially within the context of collaborative learning. Usual approaches to such issues provide their systems with a kind of expertise using a set of heuristics and domain theories built in the procedures (programming languages). This means that the programmers, not the systems, have an understanding of the knowledge being used. As a result, these systems cannot share or build new knowledge, ignore the existence of theories on which the knowledge is based, and cannot justify their recommendations systematically and scientifically [15].

To develop an IES that supports collaborative learning (CL) is especially challenging in view of knowledge representation. Current knowledge concerning CL is based on various learning theories, which are always expressed in natural language and are particularly complex given the context of group learning where the synergy among learner's interactions affect the learning processes and hence learning outcome. It is, in fact, currently difficult for both humans and computers to clearly understand and differentiate between the various learning theories. However, without their explicit representation, it is difficult to support group activities based on well-grounded theoretical knowledge.

The use of ontological engineering and ontologies for knowledge systematization have shown significant results to bridge the deep conceptual gap between how to represent the knowledge of educational environments, considering educational theories, and how to use it adequately [2; 15]. In practical terms, ontological engineering helps to achieve [4]: (a) a common vocabulary and highly structured definition of concepts; (b) semantic interoperability and high expressiveness; (c) coherence and systematization of knowledge; and (d) meta-models and foundations for solving different problems in a variety of contexts.

The main problem we are addressing is how to propose effective and pedagogically sound CL sessions supported by learning theories to facilitate the design and analysis of groups' activities and later the re-formation of groups based on previous information about successful/unsuccessful group formations and learners' interactions 
(accumulation of knowledge). Our work intends to support the selection of appropriate set of learning theories and their characteristics offering (a) fundamental settings to design group activities and to assign roles and strategies for each learner; and (b) essential conditions to analyze learners' interactions facilitating the prediction of benefits in the learning process. The design of a CL session is a requisite to maximize educational benefits and to minimize the load of interaction analysis.

To fulfill our goals, in this paper we intend to present an ontological framework to represent learning theories that support CL. Thus, we can establish a common understanding about what a learning theory is by representing it in terms of its explicit and formalized concepts and vocabulary. Then, we propose techniques to reasoning on these theories, which contribute to solving the problem of selecting an appropriate set of learning theories to propose effective learning groups. And finally, we present a prototype of a CL design tool called CHOCOLATO that uses our framework to facilitate the design of well thought out CL sessions.

\section{Background and Related Work}

Collaborative learning has a long history in Education [5]. In spite of that, to propose effective CL sessions and to capture what really happens in each session still are very complex issues due to a lack of comprehensible models for representing what is occurring [8]. Current research focuses on technological aspects rather than pedagogical aspects. Some researches have focused on how to develop programs to automate some steps of collaborative learning (ex. group formation) $[12 ; 13]$. Others propose programs to automate the use of common practices used in classroom [6; 17]. However, none of these studies consider the effectiveness of CL, allowing the design of group activities that identify adequate roles and goals for learners and match those with feasible interactions. Such link between roles, goals and interactions are essential to support the achievement of educational benefits [8]. Our initial intention is not to automate, but to provide theoretical frameworks and models to form a group on which we can validate and justify the effectiveness of our recommendations. Our goal is to improve group formation, the design of CL activities, and the analysis of learner's interactions supported by theories that have already been extensively evaluated by the Learning Science Community.

Many learning theories contribute to in-depth understanding and the support of collaborative learning (for instance, peer tutoring, anchored instructions, etc). However, it is not easy to find models that allow explicit representation of these theories. One of the reasons is the difficulty in understanding the theories due to their complexity and ambiguity. Different theories can describe the same situation using different terminologies. Moreover, each theory has its own point of view, learning focus, structure, and many other aspects that need to be considered. Therefore, to provide systems with theoretical knowledge for collaborative learning we must: a) establish a common conceptual infrastructure on which we can build a model that describes what a learning theory is and what collaborative learning is; b) clarify how learning theories can help the design of group activities and enhance learning outcomes; and c) support theory-driven group formation that facilitates the design of CL activities and analysis of interactions.

Usually the design of CL activities and analysis of interactions are treated separately. There is a clear boundary between them. To improve the design, most researchers focus on developing a set of instructions specifying how learners in a group should interact and collaborate in CL sessions (also known as CSCL scripts) [14]. Conversely, to understand the effect of interactions in the learning process, many researchers have been analyzing how learners construct their knowledge through social interactions [18]. Because of this division there are only a few researches addressing how design of CL activities can guide the analysis of interactions. Furthermore, there is a lack of research addressing the influence of group formation in the design of CL activities and its impact on the CL process.

In this context our research aims at using ontologies to enable theory-driven group formation that links the design of CL activities with the analysis of interaction processes. This approach allows identifying intended goals, roles, and strategies for a group and its members during the design process. Thus, it is possible to suggest interaction patterns that can lead learners to achieve desired goals. Then we can more easily analyze individuals' and group's interactions to identify if the proposed interactions were carried out successfully or not and if learners attained the expected benefits (goals) or not (Figure 1).

Past achievements have successfully applied ontologies to solving some problems in CL. In 1997 Ikeda et al. [7] investigated agents' negotiations for opportunistic group formation. Inaba et al. [8; 9] have been formalizing how to represent $\mathrm{CL}$ through ontologies and proposing models to represent and analyze learners' development. Other works deployed ontologies in CSCL environments to adapt content [19], to support collaboration in real scenarios [3] and to facilitate content retrieval [20]. With these achievements it is possible to some extent to identify which kind of collaboration occurs in a CL session, to partially understand group's interactions, and to deploy ontologies in real CSCL environments. Nevertheless, there remain some limitations: (a) there is no explicit relation among interaction 
patterns and learner's development; (b) it is not easy to determine which learning theory is appropriate for explaining the learner's development through a set of events; and (c) it is difficult to propose activities in compliance with the theories to enhance interactions among learners and lead them to achieve desired goals.

The contribution of our research has been to solve these limitations by re-analyzing several learning theories, clarifying their characteristics and relationships. Then, we propose ontologies and a model to describe learning theories for CL. Finally, we summarize the achievements by providing sophisticated methods and systems to support effective group formation and discuss the future research plan for the design of CL activities and the analysis of learners' interactions based on well-grounded theoretical knowledge.

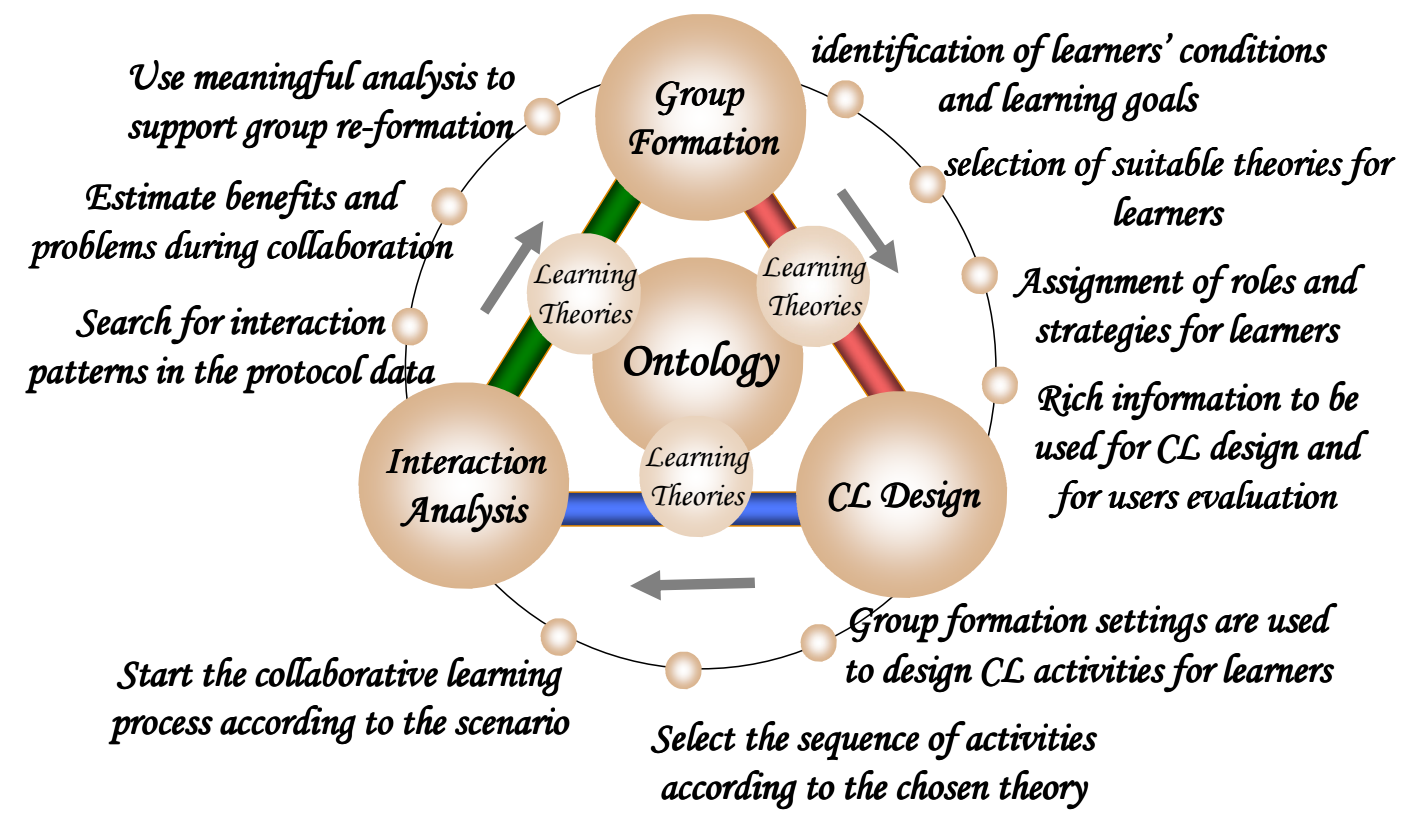

Figure 1: Ontological structure to represent a learning theory

\section{Ontology-based Framework for CL}

To understand and to clarify the characteristics of learning theories, we call upon techniques of ontological engineering to re-analyzed seven different learning theories frequently used to support CSCL activities: Cognitive Apprenticeship, Anchored Instruction, Peer Tutoring, Cognitive Flexibility, LPP (Situated Learning), Socio-Cultural Theory, and Distributed Cognition (further information about the theories can be found in [10]). Furthermore, to build our ontology we rely on the previous work of Inaba et al. [8], which proposes interaction patterns for learning theories, offers vocabulary and expected interactions' flows to describe interaction processes, and clarifies the use of theories in CL scenarios.

To comprehend each interaction in an interaction pattern, and its potential benefits for learners, we divided the interaction process in two events: instructional and learning. Every instructional event has a reciprocity relationship with the learning events. In other words, during the teaching-learning process, when a person speaks, the other listens; when someone asks a question, the other answers; and so on. Each event has a corresponding action (or actions) and possible educational benefits to the initiator of the action. These actions and educational benefits are directly related to the context (learning theory and learning strategy) in which the events are executed. We called this intrinsic relationship between instructional events and learning events influential I_L event (Figure 2b). The Influential I_L events are useful to describe the benefits of interactions in the learning process [10; 11].

The representation of the conceptual structure of a learning theory consists of two main parts (Figure 2a): the Learning Strategy and the Teaching-Learning Process. In our ontology, learning strategies are defined as $Y<=I$-goal which means how the learner who is playing the I-role will interact with another learner who is playing the role You-role. This strategy aims to support the learner who plays the I-role to achieve his individual goals (I-goals). For instance, in the Cognitive Apprenticeship theory a learner interacts with other learners to guide them during the resolution of a problem. In this case the learning strategy ( $\mathrm{Y}<=\mathrm{I}$-goal) used by this learner is "learn by guiding"; his role (I-role) is known as a "master role", the role of the learner who receives the guidance (You-role) is known as an "apprentice role", and the goals of the learner who guide (I-goal) are to acquire cognitive skills (and meta-cognitive 
skills) at an autonomous level. Previous works of our group $[8 ; 9 ; 10]$ show the strategies (Y<= I-goal), learner's roles (I-role and You-role), and individual goals (I-goal) of different learning theories. A learning theory can possibly have one or more learning strategies and also many ways of conducting the teaching-learning process (represented as “*” in Figure 2).

The Teaching-Learning Process specifies the interaction pattern of a learning theory represented by the necessary and desired interaction activities (processes) among two people (for instance, instructor and apprentice). As mentioned before, we can describe interactions using the influential I_L event for explicitly representing the interaction and its benefits from both points of view: for those who do the action and for those who receive the action. Thus, based on the analyses in Figure 2 we show our proposed ontological structure to describe a learning theory.
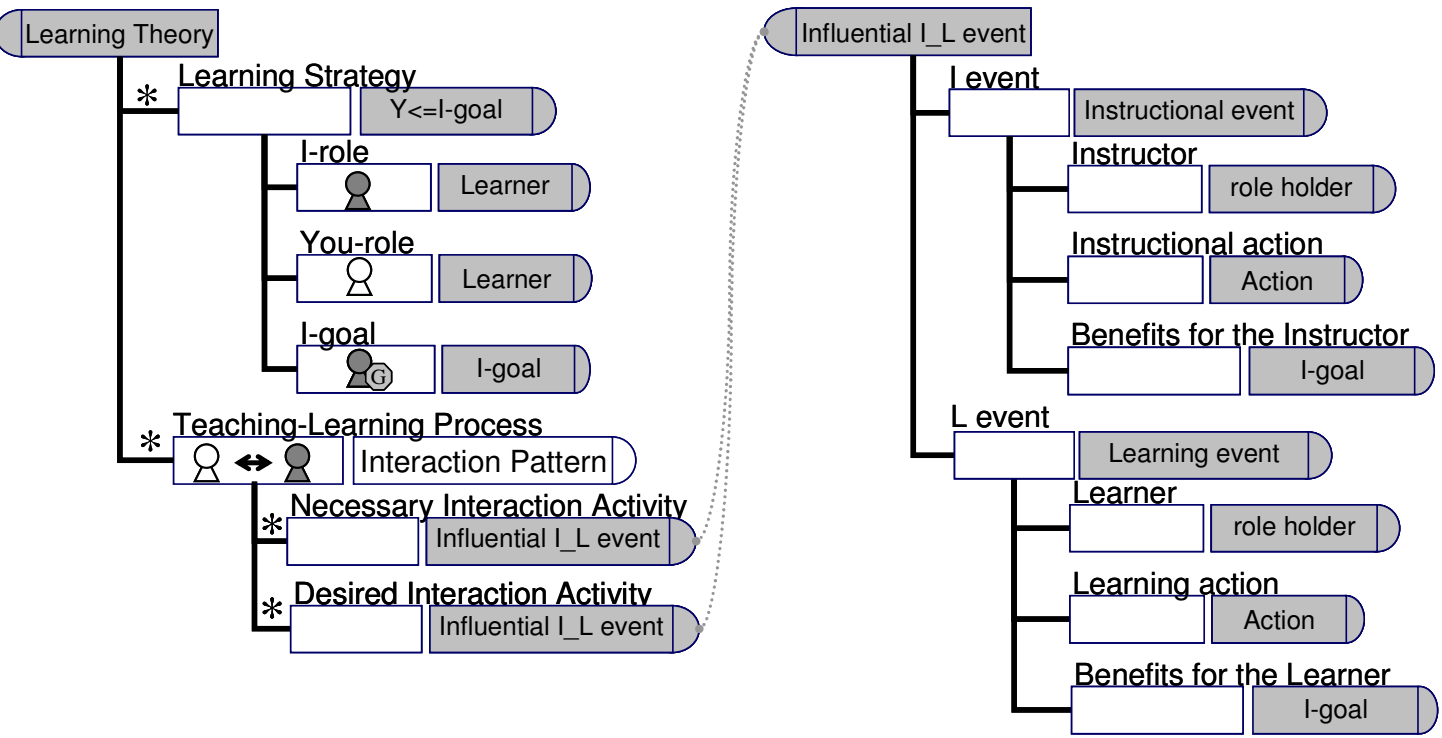

Figure 2: Ontological structure to represent a learning theory [7]

\subsection{Graphical Representation of Learning Theories}

With the representation of interaction patterns through I_L events and using our structure of a learning theory, we can identify the interaction activities and their benefits for instructor and learner in the context of a learning theory. At present, we identified more than 13 influential I_L events and their respective benefits used by the seven learning theories presented in the beginning of section 3. Thus, to facilitate the visualization by user and the reasoning by computer, we proposed the Growth Model Improved by Interaction Patterns (GMIP) [10]. It was built upon two successful previous works of Inaba et al. [10;11]. With the GMIP we clarified how learning strategies prescribed by learning theories can help learners to acquire desired goals and explicitly identify the relationships among interactions, learning strategies, and learning goals.

The GMIP is a graph model based on our ontological structure to describe an excerpt of learning theory. It represents, in a simplified way, the learner's knowledge acquisition process in compliance with the work of Rumelhart and Norman [16], and skill development process in compliance with the work of Anderson [1]. To explain the representation of our model and its compliance with the works [1] and [16], we have to explain more about two processes: knowledge acquisition and development of skills.

The process of acquiring specific knowledge includes three qualitatively different kinds of learning: accretion, tuning, and restructuring [16]. Accretion is to add and interpret new information in terms of pre-existent knowledge. Tuning is to understand knowledge through application of this knowledge in a specific situation. Restructuring is to consider the relationships in acquired knowledge and thus to rebuild the existent knowledge structure.

Considering the development of skills, there are also three phases of learning: the cognitive stage (rough and explanatory), the associative stage, and the autonomous stage [1]. The cognitive stage involves an initial encoding of a target skill that allows the learner to present the desired behavior or, at least, some crude approximation. The associative stage is the improvement of the desired skill through practice. In this stage, mistakes presented initially are gradually detected and eliminated. The autonomous stage is one of gradual continued improvement in the performance of the skill. 
Using these concepts, the GMIP graph has twenty nodes (Figure 3), which represent the levels of the learner's development at a certain moment of learning. Each node is composed by two triangles. The upper-right triangle represents the stage of knowledge acquisition, while the lower-left triangle represents the stage of skill development. The nodes are linked with arrows that show possible transitions in compliance with [1] and [16]. $s(x, y)$ is the simplified form of representing nodes in our model: $x$ represents the current stage of skill development and $y$ represents the current stage of knowledge acquisition. For instance, $s(0,0)$ represents the node where the stage of skill development and knowledge acquisition is nothing; and $s(0,1)$ represents that the stage of skill development is nothing and the stage of knowledge acquisition is accretion.

Using the GMIP graph, we show the benefits of learning theories/strategies by highlighting their path on the graph and associating each arrow with the interactions. In Figure 3 we show an example of the GMIP graph for the learning strategy "learning by apprenticeship" used by the learning theory "Cognitive Apprenticeship". Bold arrows represent the transition from one stage to the other, which is facilitated through this learning strategy using the labeled interactions. There are two kinds of interactions: the necessary interactions, represented by a black circle, and the complementary interactions, represented by a white circle. The interactions are linked by ellipses. The dashed ellipse represents a directed link between two interactions and the full ellipse represents a cyclical link between two interactions.

The GMIP clarifies more precisely how interactions proposed by theories can affect learners' development, facilitating theory-driven group formation and learning design/analysis based on learners' goals and events. Thus, our model plays a central role in the decision making about how, when, and why we should use theories to form a group considering personal and social (group) goals.

For users the GMIP allows a graphical visualization of theories and their characteristics. Thus, users can quickly interpret the theories and propose group formation and sequence of activities in compliance with them. Finally, it also gives parameters that help to measure the quality of the interaction among learners. For computers, GMIP provides a formal structure based on ontologies, which allows systems to reasoning about the theories and the features (actions, roles, strategies, etc.) prescribed by them. Thus, it becomes a powerful tool, helping designers to form a group and to select events (interactions) and roles for learners, considering interaction patterns and learning strategies appropriate for desired learning goals (and vice versa).
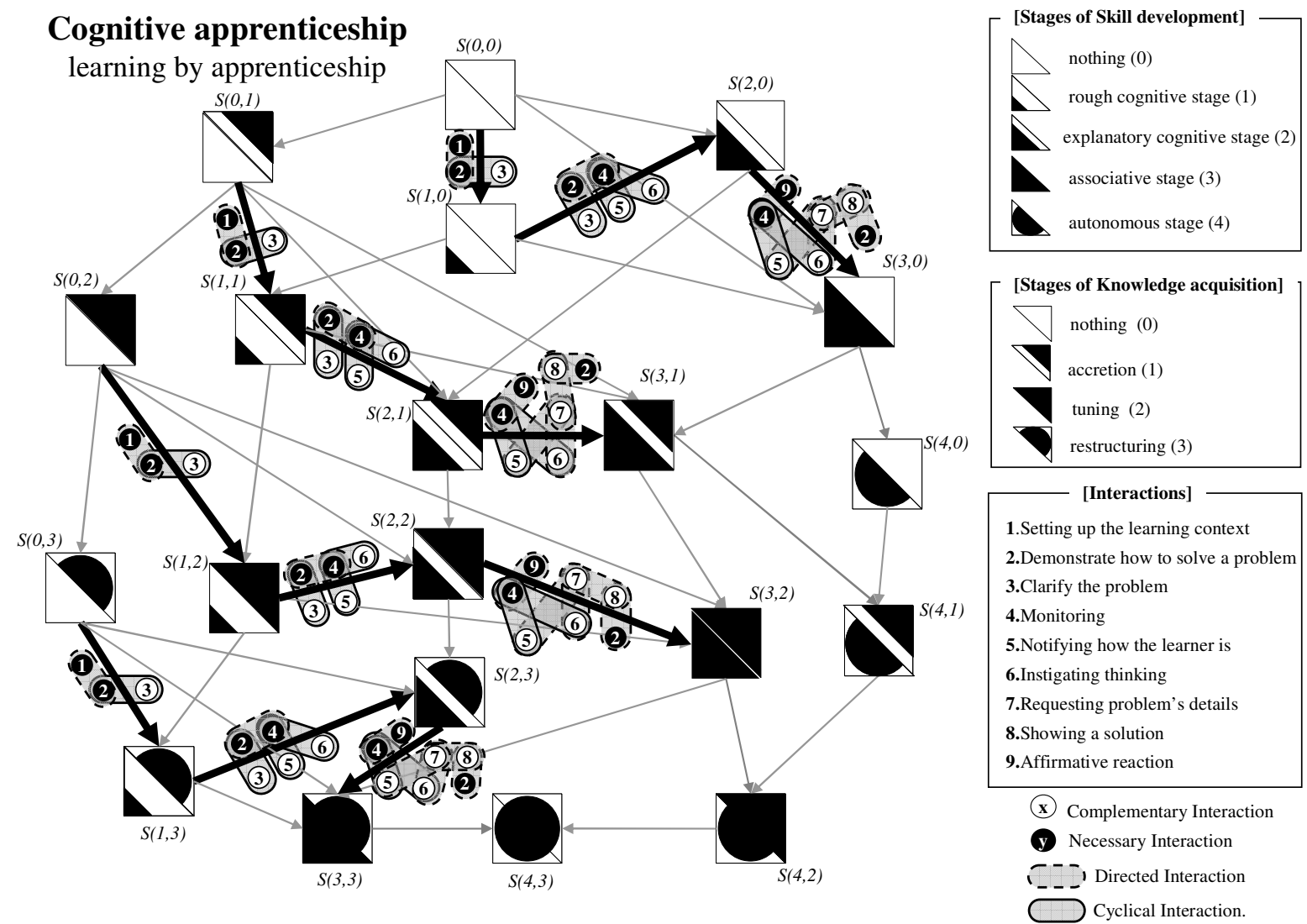

Figure 3: Example of GMIP for learning by apprenticeship used by Cognitive Apprenticeship 


\section{Towards a Complete Ontology-aware Authoring System for CL}

As we mentioned before to propose a group formation there are many learning theories such as Anchored Instruction, Peer Tutoring, Cognitive Apprenticeship, etc. Then, to assign roles and strategies for members of a group we can select appropriate set of learning theories considering the necessary pre-conditions of learners and the educational benefits we expect to be improved for each learner in the end of a CL session. This flexibility of choosing different learning theories can therefore provide us with many ways to design and conduct learning processes. However, it also

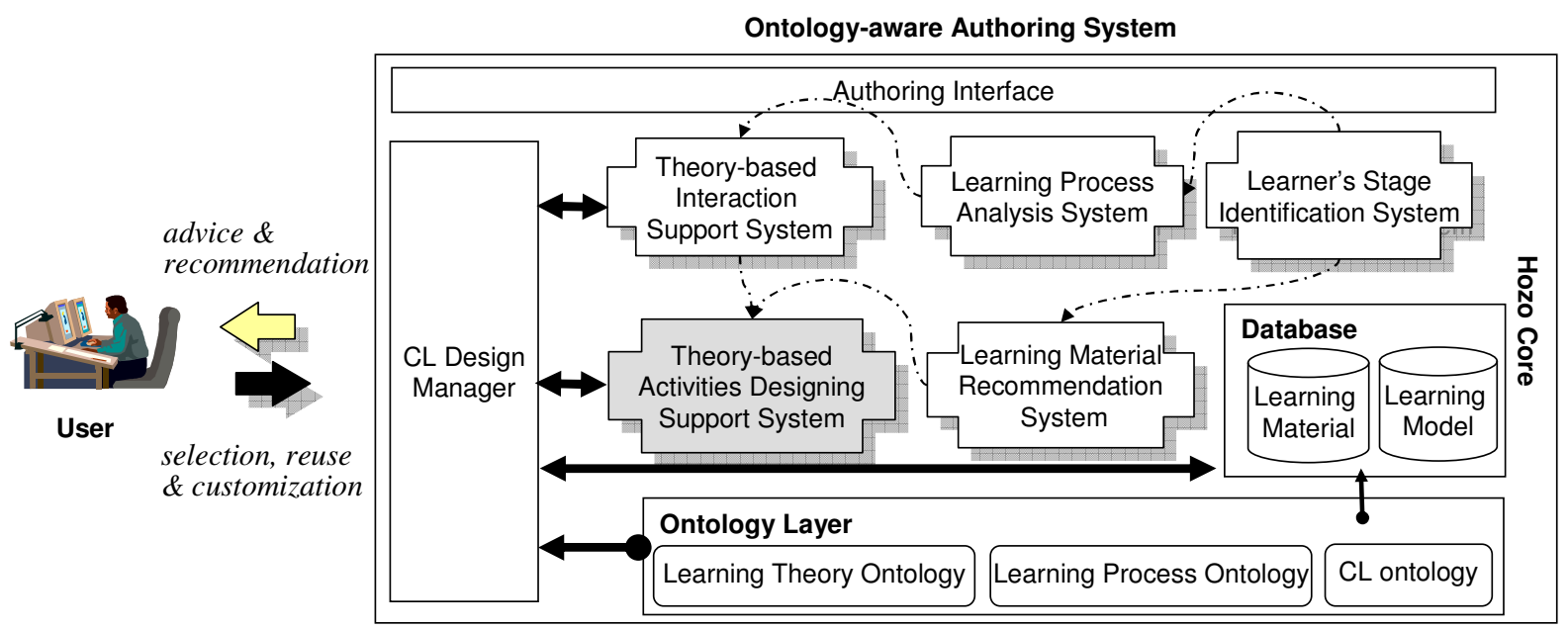

Figure 4: Architecture of CHOCOLATO

suggests the difficulty of selecting the appropriate set of learning theories during the instructional design to ensure learners' benefits and the consistency of learning processes. Therefore, to help users (instructors, teachers, designers, etc) to design effective group activities we need an elaborated authoring system that considers different learning theories to support the design in compliance with them.

To solve this problem we have been developing a theory-aware authoring system for CL, called CHOCOLATO - a Concrete and Helpful Ontology-aware Collaborative Learning Authoring Tool. It is based on our model GMIP and the ontological structure to describe learning theories, besides previous achievements presented in section 3. Through the use of ontologies, the theories and their features are declaratively and formally represented which (a) prevent unexpected interpretations of the theories; (b) provide a common vocabulary to describe them; (c) enable us to share and accumulate the knowledge; and (d) provide enough information for computational semantics to provide assistance for users based on theories. Furthermore, through the use of GMIP the system offers graphical and textual support for users providing "intelligent" guidance with theoretical justifications during the authoring process.

The architecture of CHOCOLATO is shown in Figure 4. It is proposed to support different levels of guidance during (a) group formation that maximize the educational benefit considering the individual and group goals; (b) designing of CL activities; (c) recommendation of learning materials; (d) analysis of individual and group outcomes minimizing the difficulties during this process; and (e) proposing new CL sessions based on previous group's outcomes.

This system assists both novice and expert users. For example, during the design process, for novice users, the design manager of CHOCOLATO provides a structured guidance considering different learning theories. Through an authoring interface using the GMIP it allows users to set initial conditions and goals for a learner or the group and the system automatically recommends theories, strategies, roles and activities to be performed by learners to achieve the desired goals. Furthermore, users can customize the recommendations in order to satisfy requirements depending on particular situations. For expert users, it offers a common language and guidelines to formally express CL activities, the interactions' flows learner's roles, strategies and benefits for learners. Thus, it is possible to describe new strategies and roles for learners, reuse and share them, and finally combine sequence of interactions to fit in different scenarios.

Considering the interaction analysis, it is difficult to know when learners acquire the desired benefit because we need to capture what roles the learners played and what kind of interactions occurred in the session. To help such process, the analysis system of CHOCOLATO identify when a CL session proceeds conform the initial scenario 
designed by the user. Thus, we can predict whether the learners interacted as expected and whether the CL session was successful or not. It is worth to point out that if the initial scenario of a CL session is not established previously it is much more difficult to expect concrete benefits and to analyze (quantitatively and qualitatively) how much benefits were attained by learners.

Thus, focusing on the design process, in order to produce effective CL sessions, we would like to present a sub-system of CHOCOLATO (shaded block in Figure 4) used to support the design of CL activities. This sub-system is called MARI - Main Adaptive Representation Interface. It is an ontology-aware system that uses ontologies developed in Hozo ontology editor (http://www.hozo.jp) to provide its theoretical knowledge and represent them on the screen using the GMIP. Through the use of ontologies MARI allows high expressiveness and interoperability

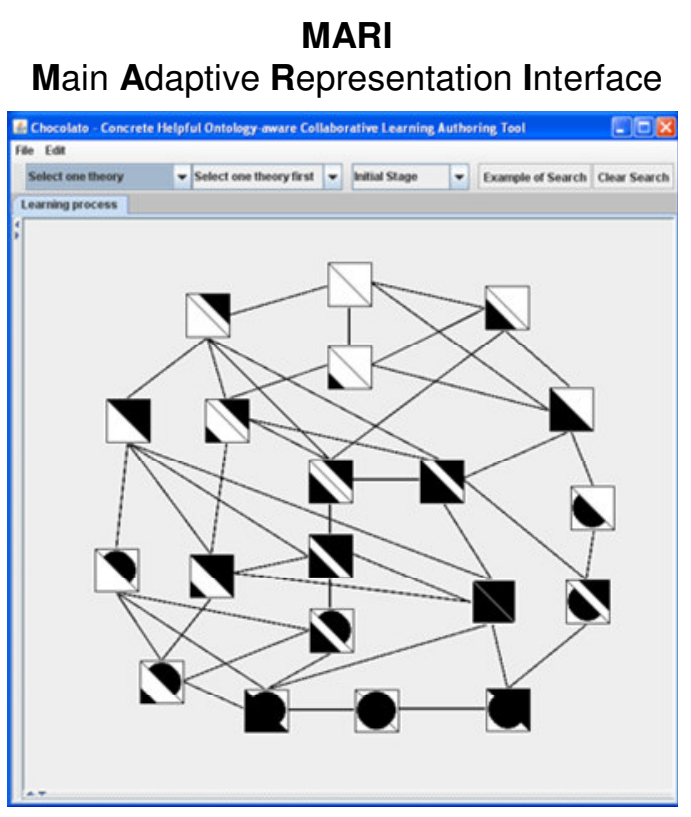

(a)

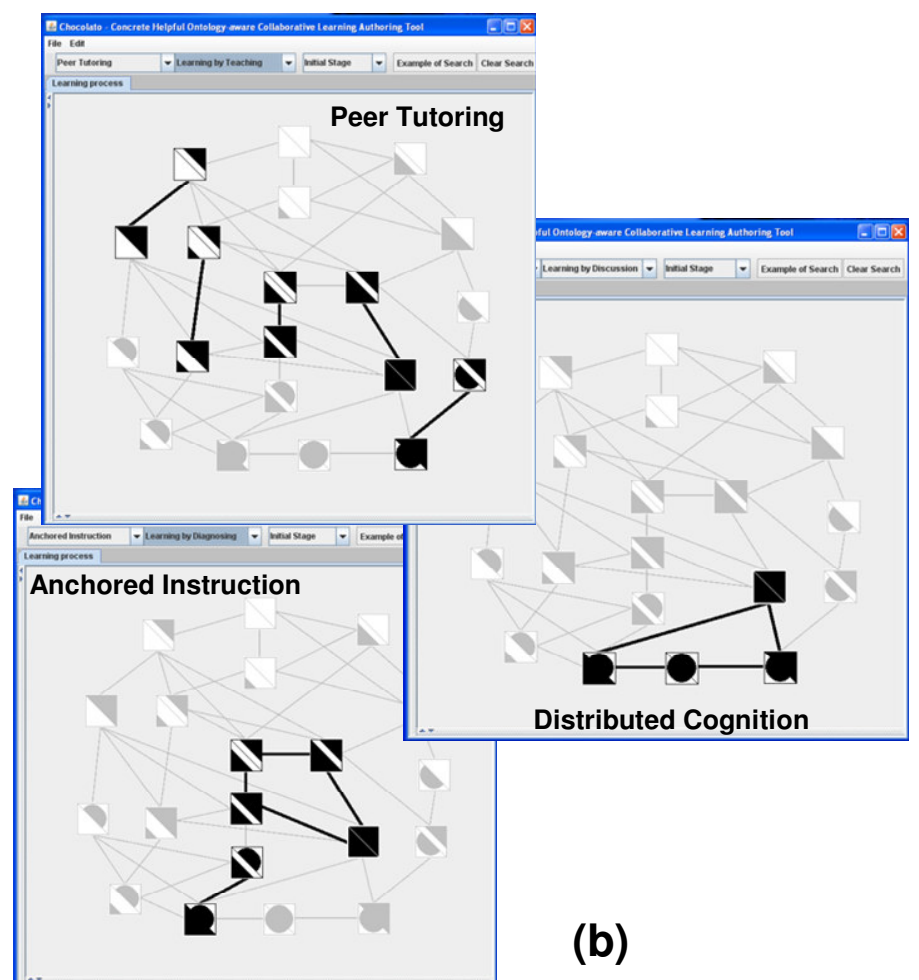

(b)

Figure 5: Graphical visualization of different learning theories and different strategies

among theories and their features. Nowadays MARI has 6 theories and 12 strategies, besides other information in its database.

MARI starts with a neutral network (Figure 5a) that can represent the theories we analyzed by selecting theories/strategies through MARI's interface. MARI reasons on the ontologies to provide a graphical visualization of them using the GMIP (Figure 5b) offering an easy and quick interpretation of necessary pre-conditions and educational benefits for learners. Furthermore, by clicking in the bold arrows the system can suggest CL activities (interactions) prescribed by the selected theory which help learners in one stage to achieve the next stage. Each interaction will be depicted on the arrows in the next version of MARI.

Another useful function in MARI is to search theories given a stage of learner's development. We can select an initial stage of a learner in the GMIP and the system will reason on the ontologies to search for any theory/strategy that has the selected stage in the beginning of the path. As same as before we can select a final stage and the system will search for any theory/strategy that has the selected stage in the end of the path. And finally, the system can search for any theory/strategy that has a path through the selected stage (it means any stage in the path). All these ways of search can be combined, thus, users can select, for example, an initial stage (pre-conditions) and a final stage (expected benefits) of a learner and the system will find the theories/strategies that help this learner to achieve the desired benefits. If more than one theory/strategy is found, users can select one of them and the system suggests activities in compliance with it.

In case we do not find any theory/strategy that helps a learner (or a group of learners) considering his initial conditions (initial stage) and desired goals (final stage), the idea of blending learning theories could be considered [11]. In such a case a possible solution to help a learner is to use the GMIP to work with theories at the macro-level (strategies, learner's stages, etc) to select a strategy $S 1$, which help learners in an stage $G 1$ (initial stage) to achieve a 
following stage $G 2$ (sub-goal), and then, to select another strategy $S 2$, which help learners in an stage $G 2$ to achieve a following stage $G 3$ (final stage) that cannot be achieved by $S 1$. After that, working with theories at the micro-level (activities, learner's roles, etc) the system is able to identify the sets of interactions of the strategies $S 1$ and $S 2$, combining them rationally, to finally, propose a sequence of CL activities that maintain the consistence of the learning process. These steps enable us to connect the strategies $S 1$ and $S 2$ and to create suitable set of interactions to help a learner in the initial condition, $G 1$, to achieve his desired goals, $G 3$.

To completely realize blended learning for CL it is necessary to consider the relationships among many assumptions described by theories (for instance, context, delivery methods, learning preferences, etc), besides the synergy among learners in a group. It is our intention for future research to include a study demonstrating some examples and possibilities to blend learning strategies semi-automatically.

Using ontologies and the GMIP it is feasible for our system to reason on the theories at the macro and micro levels and to create a link between them. This link allows us to select appropriate learning theories and strategies at the macro-level and to suggest consistent sequence of activities for learners in a group at the micro-level.

The suggestions given by our system are only guidelines for users to propose CL activities based on theories which (a) preserves the consistency of the learning process; and (b) guarantees a suitable path for learners to achieve desired benefits. However, expert designers do not need to follow the suggestions. They can propose their own path on the graph and their own sequence of activities. In such case the system also can assist these users providing different kind of information about theories, activities, strategies, learner's roles and other related information that can be useful in various situations.

\section{Conclusions}

The main contribution of this research is to introduce our model GMIP based on an ontological structure to describe learning theories for CL and create techniques to use it rationally. This is another step forward in the improvement of ontology-aware authoring systems that offer intelligent guidance to design CL activities supported by theoretical knowledge that solves, at least partially, the problems of knowledge representation presented in [15]. The proposed system MARI supported by our model GMIP and theories described as ontologies allow us to work with theories at the macro and micro levels and to create a link between them. This link clarifies, more precisely, how interactions can affect learner's development which helps designers to select interactions and roles for each learner with justifications based on the theories. It also allows us to reasoning on these theories semi-automatically to suggest consistent sequence of activities for learners in a group.

The prototype of IES, using our model and ontologies, has shown good results [11]. With principled group formation our system creates favorable conditions for learners to perform CL activities and help instructors to more easily estimate how many benefits the learners attain in the end of a session. Through an authoring interface using the GMIP, it allows users to set initial conditions and goals for learners and the system automatically recommends group formation, theories, strategies, roles and activities/interactions to be performed by learners to achieve the desired goals. Furthermore, users can customize the recommendations in order to satisfy requirements depending on particular situations. Nowadays the system (called MARI) has 6 theories, 12 strategies and 10 learner's roles, besides other information in its database.

We also would like to emphasize the intriguing possibility of blending strategies from theories using our model and our system as a feasible and novel solution to deal with the problem of unreachable goals (stages in GMIP that none of the analyzed theories has a path through by itself). Because each strategy is intrinsically represented as a path on the GMIP graph, we can find common points (stages) between strategies, and thus provide guidelines to blend learning theories by "linking" two or more strategies from different theories to achieve a desired goal. In such a case, during the CL design the system can dynamically suggest for users a set of activities supported by blended theories to find a suitable way to lead learners to achieve a desired benefits/goal.

Our ultimate objective is to complete the development of the foundations of an intelligent authoring tool for CL that supports group interactions, facilitates the design of learning environments, and evaluates the quality of learning processes. We also plan to use our achievements to allow meaningful interaction analysis to propose group formation and re-formation of groups based on an accumulation of knowledge.

\section{References}

[1] Anderson, J. R. (1982) Acquisition of Cognitive Skill. Psychological Review, 89(4), 369-406. 
[2] Aroyo, L. and Dicheva D. AIMS: (2001) Learning and Teaching Support for WWW-based Education. International Journal for Continuing Engineering Education and Life-long Learning, 11(1/2), 152-164.

[3] Barros, B., Verdejo, F., Read, T., and Mizoguchi, R. (2002) Applications of a Collaborative Learning Ontology. In Proceedings of Mexican international Conference on Artificial intelligence, 301-310.

[4] Devedzic, V. (2002) Understanding Ontological Engineering. Communications of the ACM, 45(4), 136-144.

[5] Dillenbourg, P. (1999) What do you mean by Collaborative Learning, Collaborative Learning and Computational Approaches, Oxford: Elsevier Science, 1-19.

[6] Hernández-Leo, D., Asensio-Pérez, J.I., Dimitriadis, Y. (2006) Collaborative learning strategies and scenario-based activities for understanding network protocols Proceedings of the ASEE/IEEE Frontiers in Education Conference, S2F 19-24.

[7] Ikeda, M., Go, S. and Mizoguchi, R. (1997) Opportunistic Group Formation: A Theory for Intelligent Support in Collaborative Learning. In Proceedings of the Int. Conference on Artificial Intelligence in Education, 167-174.

[8] Inaba, A., Ohkubo, R., Ikeda, M. and Mizoguchi, R. (2003) Models and Vocabulary to Represent Learner-to-Learner Interaction Process in Collaborative Learning. In Proceedings of the Int. Conference on Computers in Education, IOS Press, 1088-1096.

[9] Inaba, A., Ikeda, M. and Mizoguchi, R. (2003) What Learning Patterns are Effective for a Learner Growth?. In Proceedings of the Int. Conference on Artificial Intelligence in Education. IOS Press, 219-226.

[10] Isotani, S. and Mizoguchi, R. (2006) A Framework for Fine-Grained Analysis and Design of Group Learning Activities. In Proceedings of the Int. Conference on Computers in Education. IOS Press, v.151, 193-200.

[11] Isotani, S. and Mizoguchi, R. (2007) Using Ontologies for an Effective Design of Collaborative Learning Activities. Proceedings of the Workshop on Ontologies and Semantic Web Services for Intelligent Distributed Educational Systems, 44-53.

[12] Legras, F. and Tessier, C. (2003) LOTTO: group formation by overhearing in large teams. Proceedings of the international joint conference on Autonomous agents and multiagent systems, 425-432.

[13] Martin W. and Hans-Rüdiger P. (2001) Group formation in computer-supported collaborative learning. Proceedings of the International ACM SIGGROUP Conference on Supporting Group Work, 24-31.

[14] Miao, Y., Hoeksema, K., Hoppe, H. U., and Harrer, A. (2005) CSCL scripts: modelling features and potential use. In Proceedings of Computer Support Collaborative Learning: Learning Conference, 423-432.

[15] Mizoguchi, R. and Bourdeau, J. (2000) Using Ontological Engineering to Overcome AI-ED Problems. International Journal of Artificial Intelligence in Education, 11(2), 107-121.

[16] Rumelhart, D.E., \& Norman, D.A. (1978) Accretion, Tuning, and Restructuring: Modes of Learning, Semantic factors in cognition. LEA, 37-53.

[17] Soh, L. Khandaker, N. Jiang, H. (2006). Multiagent Coalition Formation for Computer-Supported Cooperative Learning. In Proceedings of Innovative Applications of Artificial Intelligence, 1844-1851.

[18] Suthers, D. (2006) Technology affordances for intersubjective meaning making: A research agenda for CSCL. International Journal of Computer-Supported Collaborative Learning, 1(3), 315-337.

[19] Vega-Gorgojo, G., Bote-Lorenzo, M. L., Gómez-Sánchez E., Dimitriadis, Y. A. and Asensio-Pérez, J. I. (2006) A semantic approach to discovering learning services in grid-based collaborative systems. Future Generation Computer Systems. 22(6), 709-719

[20] Yang, S. J. H., Chen, I. Y. L., \& Shao, N. W. Y. (2004). Ontology Enabled Annotation and Knowledge Management for Collaborative Learning in Virtual Learning Community. Educational Technology \& Society, 7 (4), 70-81. 